\title{
Multiplicity correlation between neutrons and gamma-rays emitted from SNM and non-SNM sources
}

\author{
Gennady Miloshevsky ${ }^{*}$, Ahmed Hassanein
}

Center for Materials under Extreme Environment, School of Nuclear Engineering

Purdue University, 400 Central Drive, West Lafayette, IN, 47907-2017, USA

\section{ABSTRACT}

The challenge in detection and identification of Special Nuclear Materials (SNM) is to discriminate between the time-correlated neutrons and gamma-rays emitted from SNM and those originating from non-correlated or differently-correlated environmental non-SNM sources. Time-correlated neutron and gamma-ray bursts can be generated by penetrating components of cosmic radiation. The characteristic features or attributes of correlated signatures can be revealed by analyzing the joint probability density functions (JPDFs) of various parameters of neutrons and gamma-rays. Monte Carlo simulations of SNM and cosmic-ray (non-SNM) sources of neutrons and gamma-rays are performed. For both SNM and non-SNM sources, energy-lifetime JPDF of neutrons, energy-lifetime JPDF of gamma-rays, and JPDFs of neutron-gamma-ray multiplicity are evaluated. Mean values, standard deviations, covariance and correlation are estimated. It is found that the number (multiplicity) of neutrons and gamma-rays emitted from an SNM source is moderately correlated $(\sim 0.48)$. The multiplicity of neutrons and gamma-rays 
generated by cosmic-ray showers at sea level is only weakly correlated $(\sim-0.046)$. The exploitation of neutron-gamma-ray multiplicity correlation in detectors can provide a tool to discriminate non-SNM sources.

Keywords: special nuclear materials, cosmic-ray radiation, Monte Carlo simulation, joint and marginal probability density functions, multiplicity, correlation

*Corresponding author. Tel: 765-494-8618, Fax: 765-496-2233, Email address: gennady@purdue.edu (Gennady Miloshevsky).

\section{Introduction}

The threat of proliferation activities and unauthorized movement of SNM cannot be underestimated [1]. The detection and identification of SNM is enormously difficult due to the complex physics laws, where background radiation, distance and time factors can severely attenuate gamma-ray or neutron signals [2]. The time course of neutrons and gamma-rays contains information that can be used to determine properties of SNM sources. For nuclear materials that support fission chains, a random event such as spontaneous fission is followed by a correlated number of neutrons and gamma-rays emitted by the fission chain [3]. These particles are emitted simultaneously and are therefore correlated in time. Recognition of these multiple particles provides a method to detect SNM. The SNM signatures can be very robust and distinct compared to natural background radiation and time-correlated cosmic-ray (non-SNM) sources [4]. Their understanding is essential for detection and identifying the presence of SNM. 
Many neutron detectors heavily rely on neutron multiplicity and time-correlation techniques [5]. Both passive and active interrogation approaches exist for searching of radiological threats. Naturally emitted neutrons and gamma-rays by SNM form the basis for all passive detection methods $[6,7]$. Active interrogation methods probe SNM by various types of radiation yielding very unique and often strong signatures $[2,8]$. However, there are many possible sources of neutrons and gamma-rays in the environment due to terrestrial, atmospheric, and cosmic-ray induced backgrounds, as well as man-made sources for industrial and medical uses $[9,10]$. About a third of the natural radiation background originates from cosmic-rays [11]. The interaction of cosmic-rays with nearby shield and metals can increase the background by many times, making the detection of fission sources extremely difficult [12]. Signatures of both gamma-rays and neutrons in normal background may be similar to those of SNM. The grosscounting detectors are unable to distinguish time-correlated particles induced by cosmic events from those emitted by a fission source [5]. Therefore, the capability of SNM detectors to use more parameters (energy, lifetime, joint neutron-gamma-ray multiplicity, etc.) can offer significant improvements in identifying the presence of SNM. The multivariate statistic of correlations in many-parameter data can be utilized in detectors to eliminate cosmic bursts of neutrons and to reduce the frequency of false alarms.

The measurements or simulations of JPDFs of various parameters of neutrons and gamma-rays emitted by SNM and non-SNM sources are not well presented in the literature. What is mainly reported is multiplicity distributions based on the analysis of the time of arrival of neutrons. Measurements and correlation simulations of neutron multiplicity distributions of spontaneous fission for a number of isotopes were performed using the COG radiation transport code [3]. It is found that even low multiplying systems with low spontaneous fission activity 
such as Highly Enriched Uranium (HEU) can be detected passively using neutron correlation techniques, and simulations can faithfully predict the physical features of fission systems. One of recent advances reported in neutron-based explosive detection is the use of two parameter (timeenergy) data that allows better signal to noise characterization and makes a wider range of signatures accessible [13]. The analytical approach was developed to describe the statistical properties of a joint number of neutrons and gamma-rays emitted from fissile samples [14]. The value of this work is an attempt to analyze the joint number statistics (probabilities of joint detections) of neutrons and gamma-rays. It was concluded that using joint detection probabilities could enhance the identification and detection of SNM samples. We have recently studied the time correlation of cosmic-ray-induced neutrons and gamma-rays at sea level [15]. The lifetime and multiplicity distributions, coincidence count statistic, and excess variance of neutrons and gamma-rays in air showers are analyzed. In most of the cosmic events, zero neutrons and one gamma-ray are observed at sea level. The energy-angle correlation for both neutrons and gamma-rays emitted from an HEU source is also recently investigated using the developed MONSOL code [16]. It is found that the energy and angle are only weakly correlated.

In this work, we have performed Monte Carlo (MC) simulations of spontaneous fission chains in an HEU source and cosmic-ray-induced background of neutrons and gamma-rays at sea level using the MONSOL code. From these MC simulations, neutron-gamma-ray multiplicity, energy-lifetime distribution of neutrons, and energy-lifetime distribution of gamma-rays are created and analyzed for both HEU and cosmic-ray sources. The marginal probability density functions (MPDFs) are also derived. Mean values and standard deviations of energy, lifetime and multiplicity of neutrons and gamma-rays are calculated. Covariance and correlation between energy-lifetime of neutrons, energy-lifetime of gamma-rays, and neutron-gamma-ray multiplicity 
are evaluated. Moderate correlation is found between multiplicity of neutrons and gamma-rays emitted from an HEU source, while this multiplicity correlation is small for cosmic-ray-induced background. The paper is organized as follows: in Section 2 we describe the computational models; in Section 3 results and discussion are presented; and conclusions are provided in Section 4.

\section{Computational models}

\subsection{Benchmarking the MONSOL code}

The MONSOL computer code [16] is used to carry out MC simulations of the transport of neutrons and gamma-rays produced from the fission of SNM and those generated by cosmicrays (non-SNM source) at sea level. The nuclear database TALYS [17] is implemented in the MONSOL code as a source of nuclear parameters, neutron and gamma-ray cross sections, nuclear reaction models, discrete energy levels, etc. These nuclear data were validated against experimental results for a large set of nuclear reactions and presented in TALYS manual [18]. The mean free paths of neutrons in different materials calculated using MONSOL and reported in ref. [16] were found to be in agreement with experimental data. The energy spectra of neutrons and gamma-rays emitted from a bare HEU sphere were calculated using the MONSOL, MCNPX and GEANT4 codes and compared [16]. These spectra are found in a good agreement

for both spontaneous and interrogation-induced fission. The cosmic-ray-shower library (CRY) [19] developed at Lawrence Livermore National Laboratory is implemented in MONSOL in order to generate distributions of energy, multiplicity, time of arrival, coordinates and direction 
of neutrons and gamma-rays at sea level. The CRY data were validated against available experimental cosmic-ray results demonstrating a very good agreement [19]. The validity of CRY data is also confirmed in recent measurements of neutron background [20] and studies of hadronic interactions of cosmic rays with materials [10]. Thus, the MONSOL modeling of neutron and gamma-ray transport is based on high-fidelity TALYS and CRY data models.

As a further validation of MONSOL code relevant to this work, the number (multiplicity) distribution of neutrons and gamma-rays emitted from a fissile source is benchmarked and compared with the results reported in ref. [14]. A plutonium $(\mathrm{Pu})$ metal sphere with a radius of $1.7 \mathrm{~cm}$, composition of $20 \%$ of ${ }^{240} \mathrm{Pu}$ and $80 \%$ of ${ }^{239} \mathrm{Pu}$, and mass density of $15.9 \mathrm{~g} / \mathrm{cm}^{3}$ is used as a source of outgoing neutrons and gamma-rays. Events of nuclear fission, particle transport, multiplication and absorption are considered in a bare Pu sphere. The number distributions of neutrons and gamma-rays escaped from a sphere were calculated separately as well as jointly. The results are shown in Fig. 1. Fig. 1(a) compares the neutron number distribution calculated using MONSOL with the results obtained from the MCNP-PoliMi code [21] and computed from the analytical model [14]. The similar comparison of gamma-ray number distributions is shown in Fig. 1(b). In both cases we observe a good agreement. The emission of two neutrons and one gamma-ray from a bare $\mathrm{Pu}$ sphere are the most probable events. The probabilities of larger numbers of particles escaping from the sphere are significantly reduced. The long tails of number distributions are due to multiplication processes in fission chains. The quantitative comparison of joint neutron-gamma-ray number distributions is difficult because of 2D graph representation used in ref. [14]. A visual collation of joint number probabilities of neutrons and gamma-rays calculated using MONSOL has demonstrated a reasonable agreement with MCNP-PoliMi data. 


\subsection{Simulation set-up of SNM and cosmic-ray systems}

A bare HEU sphere with radius of $3.97 \mathrm{~cm}$ and mass density of $19.1 \mathrm{~g} / \mathrm{cm}^{3}$ is used to investigate spontaneous fission and nuclear chain reactions. The number of neutrons emitted due to spontaneous fission from $1 \mathrm{~kg}$ of HEU is about $\sim 3-4$ neutrons per second. The composition of uranium isotopes is $1.2 \%$ of $\mathrm{U}^{234}, 93.3 \%$ of $\mathrm{U}^{235}$, and $5.5 \%$ of $\mathrm{U}^{238}$. Using this composition, an uranium nucleus undergoing the spontaneous fission reaction is sampled. The location of nucleus is sampled uniformly within an HEU sphere. The number of emitted neutrons in fission events and their energy are sampled from the multiplicity distribution and the Watt energy spectrum, respectively [7]. The direction of fission neutrons is assumed uniformly distributed in space. The same procedure is used for gamma-rays [7]. Trajectories of neutrons and gamma-rays generated in nuclear chain reactions are then followed until they are absorbed or escaped from an HEU sphere. Analyzing the escaped neutrons and gamma-rays, the distribution of their lifetimes and multiplicities is constructed. The lifetime of neutrons or gamma-rays in an HEU sphere is defined as the time between a particle being born in the sphere at some point and its arrival time to the HEU sphere's surface. For each of individual spontaneous fissions, multiplicity means the frequency of simultaneous occurrence of $0,1,2$, etc. neutrons and gamma-rays at the HEU sphere surface. The multiplicity distribution is constructed as an average of multiplicities of individual fission events.

The set-up of simulation system for investigating the cosmic-ray-induced background of neutrons and gamma-rays at sea level is described in Ref. [15]. We briefly outline some features relevant to this work. For each individual air shower, lifetimes and multiplicities of neutrons and gamma-rays at sea level are sampled from the CRY library [19] and then post-processed in the 
MONSOL code [16]. The lifetime of particles in air shower is defined as the time between the particle being born in the Earth's atmosphere at some point and the time it is arrived at sea level. Multiplicity describes a probability of simultaneous arrival of certain number of neutrons and gamma-rays at sea level from cosmic air showers. This is the frequency to simultaneously

observe $0,1,2$, etc. neutrons and gamma-rays at sea level within a specified area for each of individual air showers. The simulations are carried out for latitude of $40^{\circ} \mathrm{N}$ and area size of $10,000 \mathrm{~m}^{2}$. The solar activity is set to solar minimum that corresponds to the maximum flux of cosmic-rays. The total number of sampled protons incident upon the Earth's atmosphere is $\sim 10^{10}$ corresponding to the observation time of $\sim 6.25$ days.

\section{Results and discussion}

\subsection{JPDFs and MPDFs of neutrons and gamma-rays from spontaneous fission of HEU}

The JPDFs of energy-lifetime distribution of neutrons and gamma-rays leaving a bare HEU sphere due to spontaneous fission are shown in Fig. 2(a) and (b), respectively. It can be seen in Fig. 2(a) that the JPDF of neutron energy-lifetime distribution is confined within an island. There is a small number of high-energy neutrons in the range from $\sim 0.2 \mathrm{MeV}$ to $\sim 6 \mathrm{MeV}$ with very short lifetimes ranging from $\sim 10^{-6} \mathrm{~ns}$ to $\sim 10^{-4} \mathrm{~ns}$ (shown in Fig. 2(a) as red and orange dots). In this energy range, the majority of emitted neutrons has lifetimes from $\sim 10^{-4}$ ns up to $\sim 5$ ns (yellow area in Fig. 2(a)). The next layer surrounding this yellow spot and displayed in green colors covers the neutrons with low energy (from $\sim 1 \mathrm{keV}$ to $\sim 0.2 \mathrm{MeV}$ ), with high energy (from $\sim 6 \mathrm{MeV}$ to $\sim 15 \mathrm{MeV}$ ), and those with long lifetimes between $\sim 5 \mathrm{~ns}$ and $\sim 50 \mathrm{~ns}$. It can also be 
seen in Fig. 2(a) that the lifetime of neutrons with energy $\sim 1-5 \mathrm{keV}$ is varied from $\sim 10 \mathrm{~ns}$ to $\sim 100$ ns. As the energy of neutrons increases, the range of lifetimes expands toward smaller values. For instance, for neutron energy $\sim 0.2 \mathrm{MeV}$ the lifetime range is from $\sim 0.001$ ns to $\sim 100$ ns. Neutrons with the energy $\sim 1-2 \mathrm{MeV}$ have a wide range of lifetimes from $\sim 5 \cdot 10^{-5} \mathrm{~ns}$ to $\sim 10 \mathrm{~ns}$. For higher neutron energies $>\sim 5 \mathrm{MeV}$, the range of lifetimes decreases. It is also seen in Fig. 2(a) that the fraction of neutrons with energies $\sim 0.1-10 \mathrm{MeV}$ and long lifetimes $\sim 10-100 \mathrm{~ns}$ decreases (cyan and blue areas). In this energy range, there is a small number of neutrons with lifetimes greater than $\sim 100 \mathrm{~ns}$ (blue dots). The JPDF of energy-lifetime distribution of gamma-rays (Fig. 2(b)) is different from that of neutrons (Fig. 2(a)). There is a small number of neutrons with short lifetimes ranging from $10^{-4} \mathrm{~ns}$ to $10^{-3} \mathrm{~ns}$ in the energy interval from $\sim 0.04 \mathrm{MeV}$ to $\sim 4 \mathrm{MeV}$ (red dots). The majority of gamma-rays has energies from $\sim 0.3 \mathrm{MeV}$ to $\sim 1.5 \mathrm{MeV}$ and lifetimes from $\sim 0.01 \mathrm{~ns}$ to $\sim 1 \mathrm{~ns}$ (the orange area in Fig. 2(b)). The maximum of gamma-ray lifetimes is located around $\sim 0.1 \mathrm{~ns}$. The portion of gamma-rays with lower $(<0.3 \mathrm{MeV})$ and higher $(>1.5 \mathrm{MeV})$ energies as well as smaller $(<0.01 \mathrm{~ns})$ and greater $(>1 \mathrm{~ns})$ lifetimes is significantly decreased (yellow, green and blue layers in Fig. 2(b)). A remarkable feature of the JPDF of energy-lifetime distribution of gamma-rays is the strips corresponding to spectral lines in the energy region below $\sim 0.3 \mathrm{MeV}$. They show that the lifetime of gamma-rays in spectral lines ranges from $\sim 10^{-3}$ ns to $\sim 10$ ns.

The MPDFs of energy and lifetime of neutrons are derived by integrating over one of the variables in Fig. 2(a). The MPDF of neutron energy is shown in Fig. 3(a). It is compared to the normalized energy spectra (the area under curves is unity) of neutrons calculated using MCNPX and GEANT4 codes and reported in ref. [16]. The agreement is very good, especially in the region with neutron energy $>1 \mathrm{MeV}$. The maximum of neutron energy is found near $\sim 0.8 \mathrm{MeV}$. 
For uranium isotopes, the energy spectrum of neutrons from spontaneous fission is reminiscent of a typical Watt fission spectrum [7]. The integration of JPDF in Fig. 2(a) over the energy gives the MPDF of neutron lifetime illustrated in Fig. 3(b). The MPDF of neutron lifetime shows that the number of neutrons with lifetimes $<\sim 1 \mathrm{~ns}$ is nearly constant. The fraction of neutrons with longer lifetime (from $\sim 2$ ns to $\sim 100 \mathrm{~ns}$ ) sharply drops. The calculated mean values, standard deviations, covariance and correlation of energy and lifetime of neutrons emitted from an HEU sphere are presented in Table 1. The covariance of neutron energy-lifetime relation is $\sim-1.06$ $\mathrm{MeV} \cdot \mathrm{ns}$. The correlation is $\sim-0.19$ implying that the energy and lifetime of neutrons are slightly associated.

The MPDF of gamma-ray energy represents the normalized energy spectrum of gammarays from spontaneous fission (Fig. 4(a)). The wide energy maximum is located at $\sim 0.7 \mathrm{MeV}$. For $\sim<0.5 \mathrm{MeV}$, the energy spectrum demonstrates the spectral lines that are characteristic of uranium isotopes. The number of gamma-rays with energies higher than $\sim 0.7 \mathrm{MeV}$ sharply drops. The MPDF of gamma-ray lifetime is illustrated in Fig. 4(b). It shows a broad maximum near $\sim 0.1 \mathrm{~ns}$. The number of gamma-rays with shorter lifetimes $<\sim 0.1 \mathrm{~ns}$ is slightly reduced. The number of gamma-rays with lifetimes larger than $\sim>1$ ns sharply drops. The mean values, standard deviations, covariance and correlation of energy and lifetime of gamma-rays emitted from an HEU source are given in Table 2. The covariance of the energy-lifetime relation is $\sim 0.068 \mathrm{MeV} \cdot \mathrm{ns}$. The correlation is $\sim 0.018$ that is small. The energy and lifetime of gamma-rays are positively correlated meaning that they vary in the same direction.

The JPDF of neutron-gamma-ray multiplicity is shown in Fig. 5. The most frequent event is the observation of $\sim 3$ neutrons and $\sim 1$ gamma-ray (the area colored in red). The probability of simultaneous observation of larger number of neutrons and gamma-rays emitted from a bare 
HEU sphere drops sharply (yellow, green, and cyan layers in Fig. 5). Within a statistical uncertainty, the number of observed neutrons (up to 235 ) is greater compared to that of gammarays (up to $\sim 20$ ).

The integration over one of multiplicities in Fig. 5 gives the MPDF of neutron and gamma-ray multiplicity (Fig. 6(a) and (b)). The MPDF of neutron multiplicity shows a peak near $\sim 2.5$ (Fig. 6(a)). The fission events with zero and one neutrons are also occurred frequently. The MPDF of gamma-ray multiplicity sharply drops starting from zero (Fig. 6(b)). The mean values, standard deviations, covariance and correlation of neutron-gamma-ray multiplicity are presented in Table 3. The calculated mean values of multiplicity are $\sim 2.6$ and 1.4 for neutrons and gammarays, respectively. The standard deviations are $\sim 1.6$ and $\sim 1.1$. The covariance of neutron-gammaray relation is $\sim 0.82$. The correlation is $\sim 0.48$. Thus, the multiplicity of neutrons and gamma-rays is moderately correlated.

3.2. JPDFs and MPDFs of neutrons and gamma-rays generated in cosmic air showers at sea level

The JPDF of energy-lifetime distribution of neutrons and gamma-rays produced at sea level at latitude of $40^{\circ} \mathrm{N}$ by cosmic air showers is shown in Fig. 7(a) and (b). It is seen in Fig. 7(a) that the JPDF of energy-lifetime distribution of neutrons is confined within a narrow band with its widening at higher neutron energies. The majority of neutrons that can be found at sea level are low-energy neutrons $(<\sim 1 \mathrm{keV})$ with lifetimes ranging from $\sim 0.5 \mathrm{~ms}$ to $\sim 0.5 \mathrm{~s}$. Moreover, the lifetime of neutrons decreases within a narrow band as their energy increases (Fig. 7(a)). The fraction of neutrons with energy from $1 \mathrm{keV}$ up to $10^{5} \mathrm{MeV}$ is significantly reduced 
(layers colored in yellow, green and blue in Fig. 7(a)). These neutrons have lifetimes varying from $\sim 1 \mu$ s to $1 \mathrm{~ms}$. The JPDF of energy-lifetime distribution of gamma-rays is shown in Fig. 7(b). Since the number of low-energy gamma-rays increases significantly, the gamma-rays with energies below $1 \mathrm{MeV}$ were not treated in $\mathrm{MC}$ simulations. Therefore, the part of energy-lifetime map of neutrons with energy $>1 \mathrm{MeV}$ (Fig. 7(a)) can be only compared to that shown in Fig. 7(b) for gamma-rays. It is seen that the energy-lifetime JPDF of gamma-rays is different from that of neutrons. The majority of gamma-rays arrives at sea level with energies from $\sim 1 \mathrm{MeV}$ to $\sim 100$ $\mathrm{MeV}$ and lifetimes from $\sim 0.1 \mu$ s to $\sim 100 \mu$ s (red, orange, and yellow layers in Fig. 7(b)). The fraction of gamma-rays with higher energies $(>100 \mathrm{MeV})$ is significantly decreased (areas colored in green, cyan and blue). It is seen in Fig. 7(b) that there is a population of gamma-rays with the energy from $\sim 1 \mathrm{MeV}$ up to $\sim 15 \mathrm{MeV}$ which have long lifetimes from $\sim 1 \mathrm{~ms}$ to $0.3 \mathrm{~s}$.

When the JPDF of neutron energy-lifetime (Fig. 7(a)) is integrated over lifetime, the MPDF of energy (the energy spectrum) shown in Fig. 8(a) is derived. The energy spectrum of neutrons at sea level is found in very good agreement with MCNPX results [19] and experimental measurements [22]. For comparison purposes, the MCNPX and experimental spectra were normalized such that the area under curves is equal to unity. It is seen that the energy range of neutrons at sea level at latitude of $40^{\circ} \mathrm{N}$ covers many orders of magnitude starting from thermal neutrons $\sim 0.01 \mathrm{eV}$ and ending with high-energy neutrons of $\sim 10^{5} \mathrm{MeV}$. The thermal peak near $\sim 0.1 \mathrm{eV}$ is associated with thermalization of higher energy neutrons by atmosphere's nuclei. Two other small peaks located within 1-10 MeV and near $\sim 100 \mathrm{MeV}$ are seen in the energy spectrum. The log-log scale smears these peaks. The neutrons within 1-10 $\mathrm{MeV}$ are due to the nuclear evaporation of the excited atmospheric nuclei. The origin of 100 $\mathrm{MeV}$ peak is associated with various knock-on direct nuclear reactions between high-energy 
cosmic particles and atmospheric nuclei. The MPDF of neutron lifetime in cosmic showers is illustrated in Fig. 8(b). It shows a maximum near $\sim 20 \mu \mathrm{s}$. The number of neutrons with smaller and greater lifetimes drops. The calculated mean values, standard deviations, covariance and correlation of energy and lifetime of neutrons produced by a cosmic-ray (non-SNM) source are reported in Table 1 . The covariance of neutron energy-lifetime relation is $\sim-0.08 \mathrm{MeV} \cdot \mathrm{s}$. The correlation is $\sim-0.02$. The correlation is negative and small implying that the energy and lifetime of neutrons in cosmic air showers are very weakly associated.

The MPDFs of gamma-ray energy and lifetime are shown in Fig. 9(a) and (b), respectively. They are derived as usual by integrating over one of variables in Fig. 7(b). The MPDF of gamma-ray energy (the energy spectrum of gamma-rays (Fig. 9(a)) at sea level at latitude of $40^{\circ} \mathrm{N}$ ) is a decreasing function of the energy. The spectrum is per unit energy normalized by the number of cosmic events. Since the number of low-energy gamma-rays increases significantly, the cut-off at $\sim 1 \mathrm{MeV}$ was used. The MPDF of gamma-ray energy (Fig. $9(a))$ is in a perfect agreement with the energy spectrum of gamma-rays calculated using Fluka and reported in ref. [19]. For comparison aims, the Fluka spectrum was normalized such that the total area is equal to 1.0. The MPDF of gamma-ray lifetime in cascade atmospheric showers at latitude of $40^{\circ} \mathrm{N}$ is shown in Fig. 9(b). The spectrum is averaged over the total number of cosmic events and shown in units $1 / \mathrm{s}$. The MPDF of gamma-ray lifetimes shows a maximum near $\sim 2 \mu \mathrm{s}$. This peak at $2 \mu \mathrm{s}$ is broader than that of neutrons at $20 \mu \mathrm{s}$ (Fig. 8(b)). The number of short-lived and long-lived gamma-rays is decreased. However, the gamma-ray spectrum shows a lifetime plateau within $\sim 1 \mathrm{~ms}-\sim 0.1 \mathrm{~s}$. The nearly constant amount of gamma-rays at these times could be due to delayed radiative decay processes of excited nuclei. The mean values, standard deviations, covariance and correlation of energy and lifetime of gamma-rays generated by cosmic-rays (non- 
SNM source) are presented in Table 2. The covariance of the energy-lifetime relation is $\sim-0.002$ $\mathrm{MeV} \cdot \mathrm{s}$. The correlation is $\sim-0.0055$, which is very small. The energy and lifetime of gammarays are negatively correlated meaning that they vary in different directions.

The JPDF of neutron-gamma-ray multiplicity shown in Fig. 10 is analyzed. The most probable event is the simultaneous arrival of small number of neutrons and gamma-rays (areas colored in red, orange and yellow). The probability to observe simultaneously a larger number of particles at sea level is sharply reduced. There are very rare, single cosmic air showers with a large amount of gamma-rays and neutrons (cyan strips in Fig. 10). However, the observed number of neutrons (up to $\sim 18$ ) is much smaller compared to that of gamma-rays (up to $\sim 300$ ).

The integration over one of the multiplicities in Fig. 10 gives the MPDF of neutron and gamma-ray multiplicity (Fig. 11(a) and (b)). The MPDF of neutron multiplicity (Fig. 11(a)) drops sharply starting from zero. This means that there is a significant number of cosmic air showers in which the neutrons do not reach the Earth's surface. The MPDF of gamma-ray multiplicity (Fig. 11(b)) shows a peak at one, meaning cosmic air showers with one gamma-ray at sea level are prevailing. There is also a considerable number of showers with zero gammarays. The probability to observe cosmic air showers at sea level with two and more gamma-rays sharply drops. The calculated mean values, standard deviations, covariance and correlation of neutron-gamma-ray multiplicity from a non-SNM source are reported in Table 3. The mean values of multiplicity for neutrons and gamma-rays are $\sim 0.14$ and $\sim 1.13$, respectively. The standard deviations are $\sim 0.38$ and $\sim 1.23$. The covariance of neutron-gamma-ray relation is $\sim$ 0.021 . The correlation is $\sim-0.046$ that is small.

\section{Conclusions}


The MONSOL computer code is used to calculate the joint neutron-gamma-ray multiplicity, the joint energy-lifetime distribution of neutrons, and the joint energy-lifetime distribution of gamma-rays emitted from a bare HEU source and generated by cosmic-rays at sea level. The corresponding marginal distributions of energy, lifetime, and multiplicity of these particles are also built. The simulated distributions are post-processed and analyzed in order to determine mean values, standard deviations, covariance and correlation. The degree of energylifetime correlation as well as neutron-gamma-ray multiplicity from an SNM source is compared to that from cosmic-ray-induced background (non-SNM source).

The JPDFs and MPDFs of neutrons and gamma-rays emitted from a bare HEU sphere are analyzed. The calculations are performed for the case of spontaneous fission. The absolute value of the energy-lifetime correlation of neutrons $(\sim-0.19)$ is found to be larger by an order of magnitude compared to that of gamma-rays $(\sim 0.018)$. Thus, it is observed that the energy and lifetime of neutrons are slightly correlated, but that correlation of gamma-rays is insignificant. The energy and lifetime of neutrons are negatively correlated meaning that they vary in the opposite direction, while those of gamma-rays are positively correlated meaning that they vary in the same direction. The moderate positive correlation $(\sim 0.48)$ is observed between multiplicity of neutrons and gamma-rays emitted from a bare HEU source.

The JPDFs and MPDFs of neutrons and gamma-rays in cosmic air showers representing a non-SNM source are analyzed. It is observed in general that different variables such as energylifetime and neutron-gamma-ray multiplicity are only weakly correlated. The absolute value of energy-lifetime correlation of neutrons $(\sim-0.02)$ is found to be larger by about an order of magnitude compared to that of gamma-rays $(\sim-0.0055)$. However, these values of energy- 
lifetime correlation are small. The correlation of neutron-gamma-ray multiplicity in cosmic air showers is also small $(\sim-0.046)$. Both JPDFs of energy-lifetime and neutron-gamma-ray multiplicity are negatively correlated meaning that they vary in the opposite direction.

On one side, the moderate positive correlation $(\sim 0.48)$ is found between multiplicity of neutrons and gamma-rays emitted from an HEU source. On the other side, the multiplicity of neutrons and gamma-rays at sea level in cosmic air showers is only weakly correlated $(\sim-0.046)$. Therefore, it is very difficult to observe simultaneously the burst of neutron and gamma-ray counts in detectors from a non-SNM source. The deployment of multiplicity correlation between neutrons and gamma-rays in detectors is a very promising technique. It opens the possibility for simultaneous detection of correlated neutrons and gamma-rays from SNM samples, automatically discriminating cosmic-ray-induced (non-SNM) neutrons and gamma-rays, since they are uncorrelated.

\section{Acknowledgments}

This work is sponsored by the U.S. Department of Energy (DOE), National Nuclear Security Administration (NNSA), Office of Proliferation Detection (NA-221) under grant No. DE-NA0000533.

\section{Figure captions}

Fig. 1. Comparison of the number distribution for neutrons (a) and gamma-rays (b) emitted from a bare Pu metallic sphere. (For interpretation of the references to color in this figure legend, the reader is refered to the web version of this article). 
Fig. 2. JPDF of energy-lifetime distribution of (a) neutrons and (b) gamma-rays emitted from a bare HEU sphere due to spontaneous fission.

Fig. 3. MPDF of (a) energy and (b) lifetime of neutrons emitted from a bare HEU sphere due to spontaneous fission. MPDFs of neutron energy calculated using MONSOL, MCNPX and GEANT4 codes are compared.

Fig. 4. MPDF of (a) energy and (b) lifetime of gamma-rays emitted from a bare HEU sphere due to spontaneous fission.

Fig. 5. JPDF of neutron-gamma-ray multiplicity from spontaneous fission in a bare HEU sphere.

Fig. 6. MPDFs of (a) neutron and (b) gamma-ray multiplicity from spontaneous fission in a bare HEU sphere.

Fig. 7. JPDFs of energy-lifetime distribution of (a) neutrons and (b) gamma-rays in cosmic air showers at sea level at latitude of $40^{\circ} \mathrm{N}$.

Fig. 8. MPDFs of (a) energy and (b) lifetime of neutrons in cosmic air showers at at sea level at latitude of $40^{\circ}$ N. MPDF of neutron energy is compared to MCNPX and experimental data. 
Fig. 9. MPDFs of (a) energy and (b) lifetime of gamma-rays in cosmic air showers at at sea level at latitude of $40^{\circ} \mathrm{N}$. MPDF of gamma-ray energy is compared to Fluka data.

Fig. 10. JPDF of neutron-gamma-ray multiplicity in cosmic air showers at at sea level at latitude of $40^{0} \mathrm{~N}$.

Fig. 11. MPDFs of (a) neutron and (b) gamma-ray multiplicity in cosmic air showers at at sea level at latitude of $40^{\circ} \mathrm{N}$.

\section{Table captions}

Table 1. Mean values, standard deviations, covariance and correlation of energy and lifetime of neutrons emitted from SNM and non-SNM sources.

Table 2. Mean values, standard deviations, covariance and correlation of energy and lifetime of gamma-rays emitted from SNM and non-SNM sources.

Table 3. Mean values, standard deviations, covariance and correlation of the multiplicity of neutrons and gamma-rays emitted from SNM and non-SNM sources. 

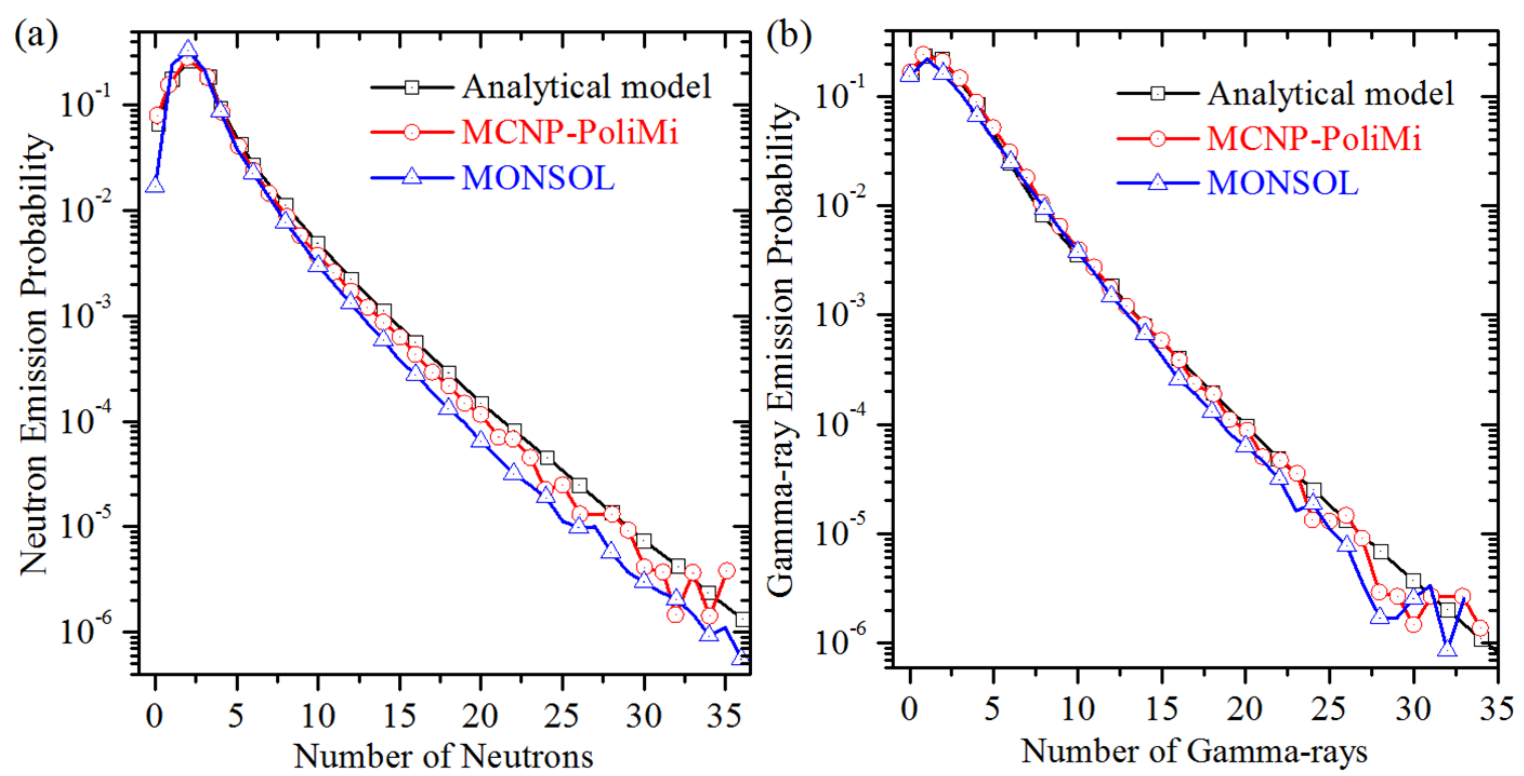

Fig. 1 

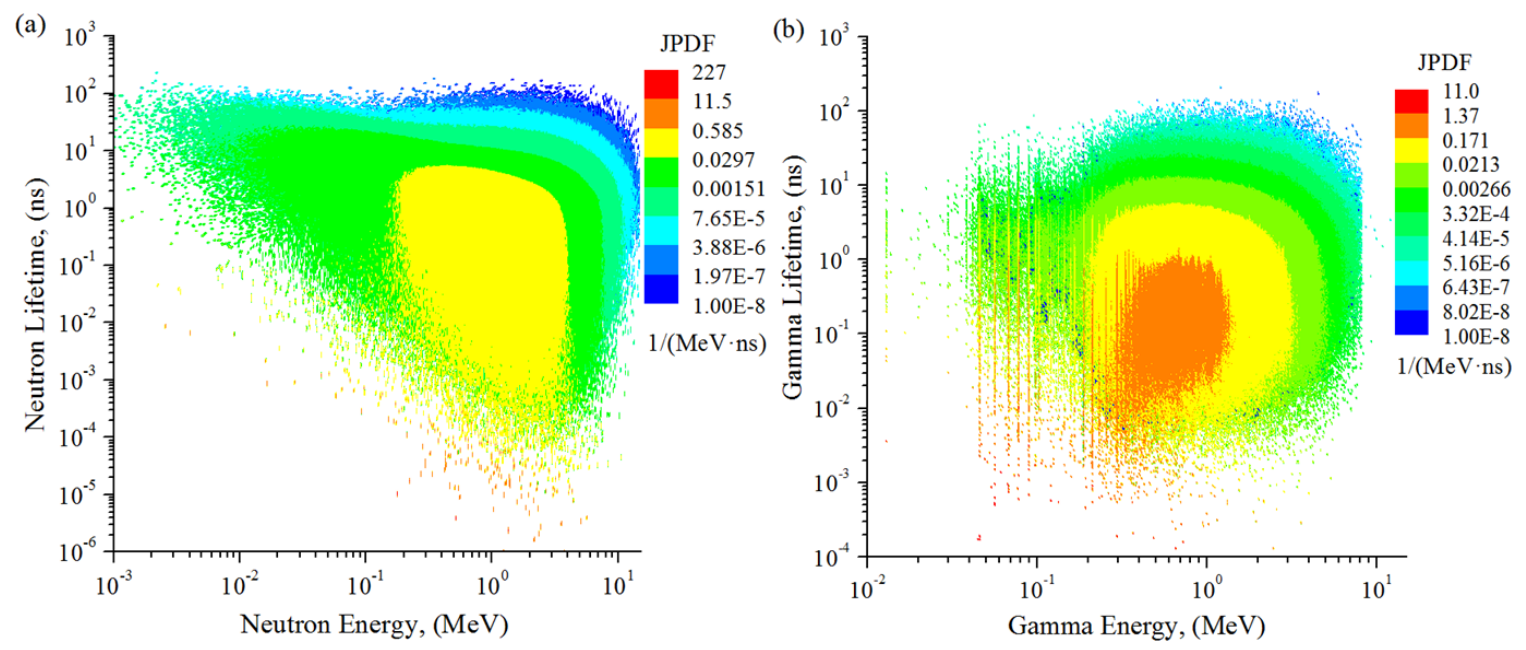

Fig. 2 

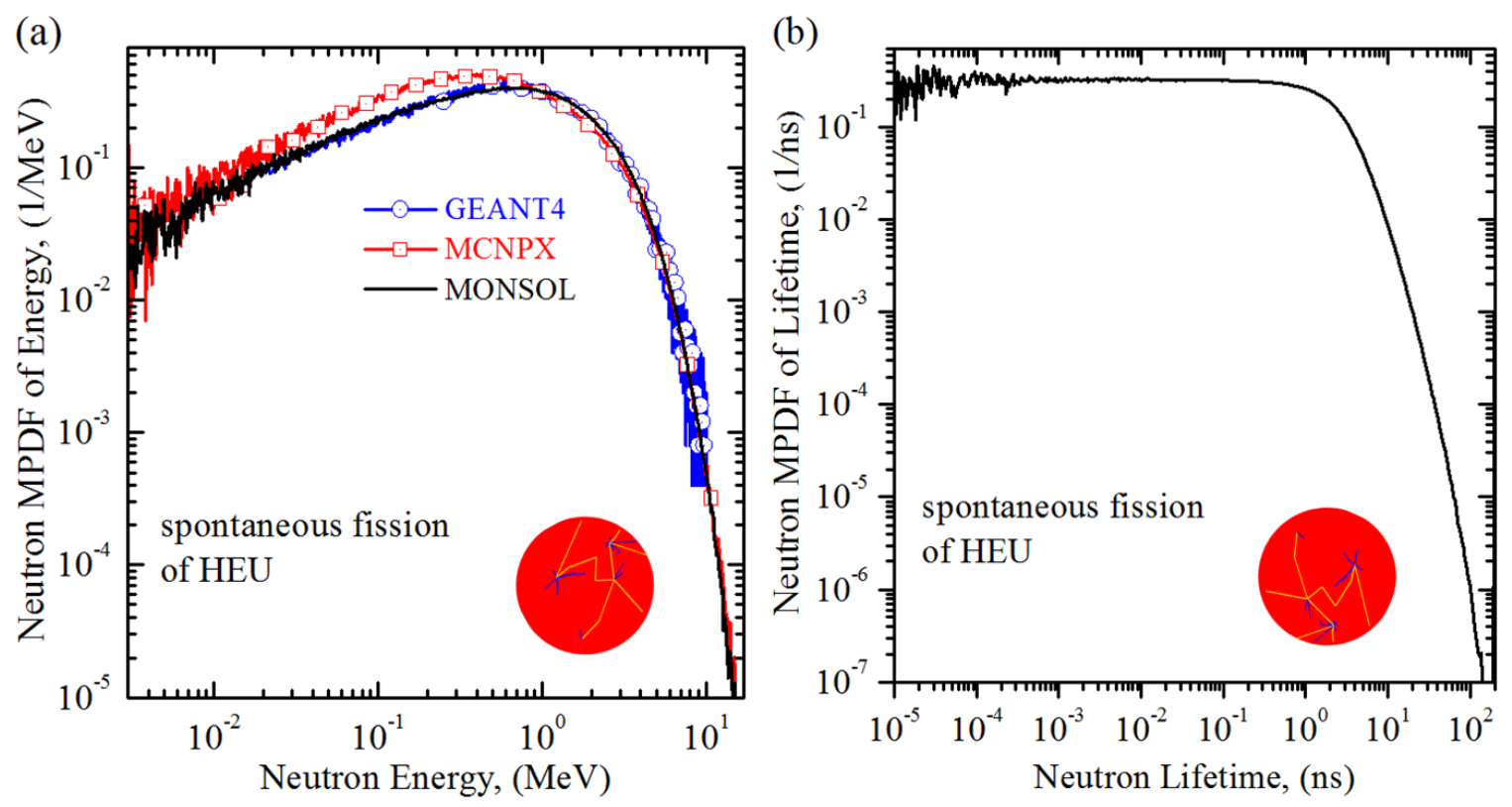

Fig. 3 

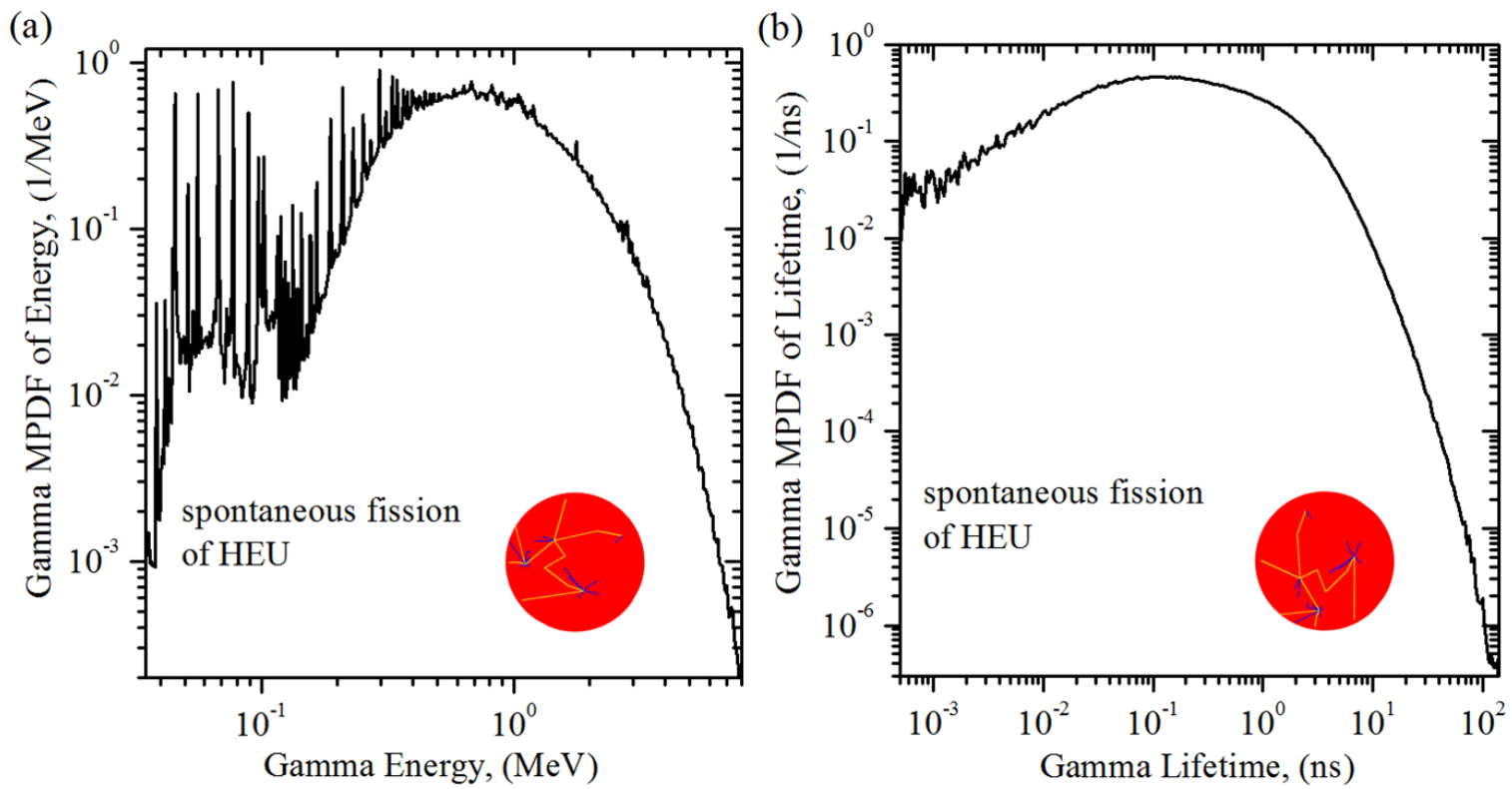

Fig. 4 


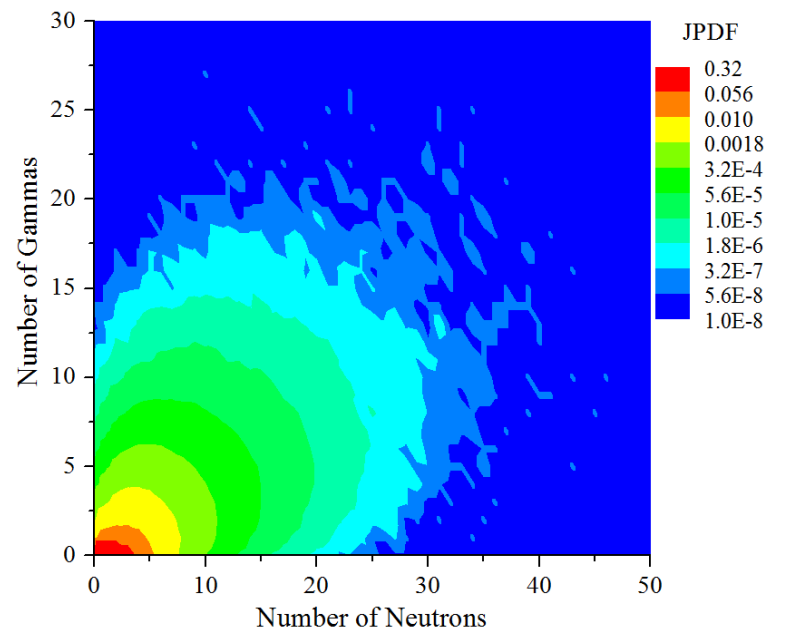

Fig. 5 

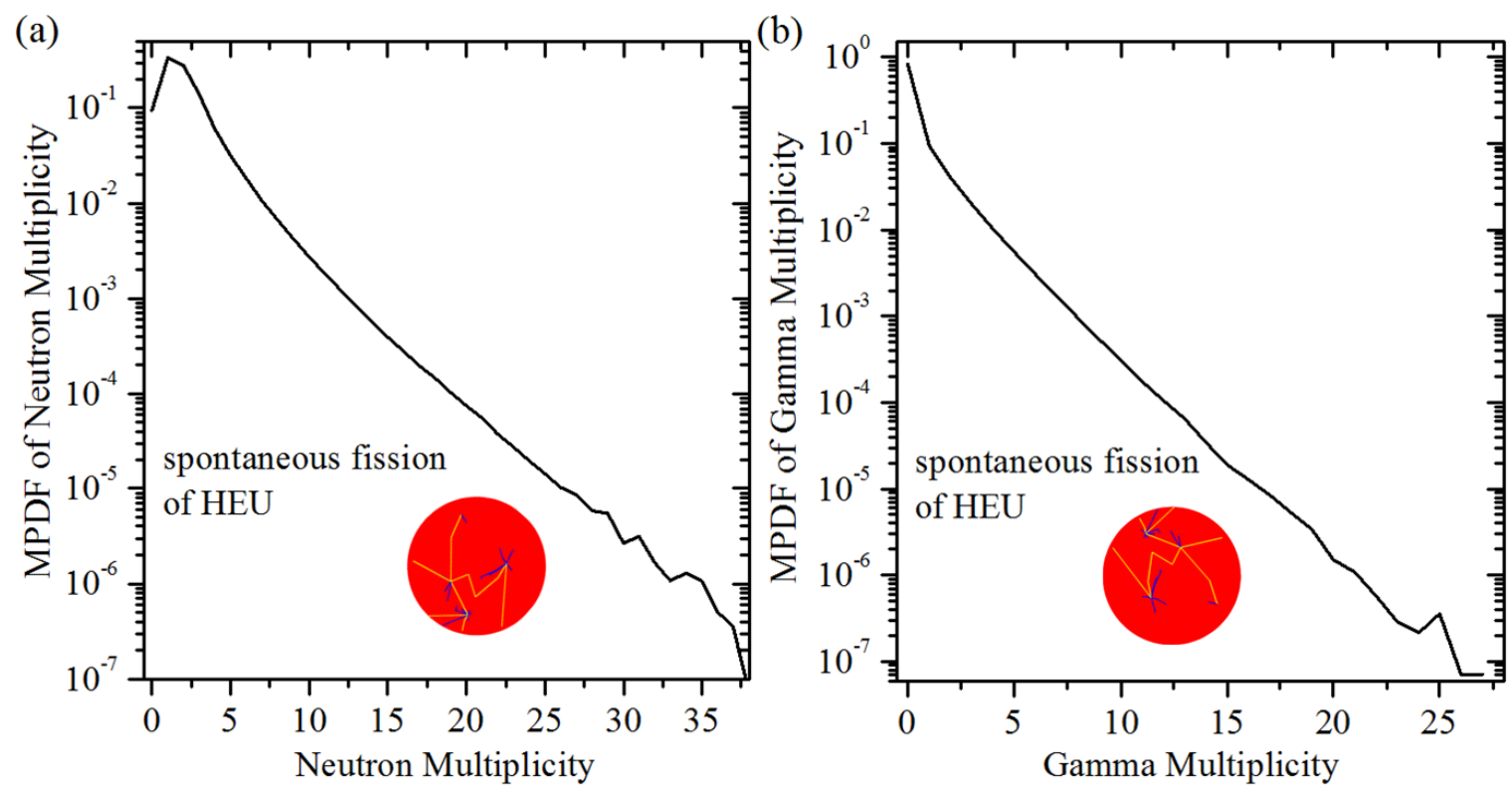

Fig. 6 

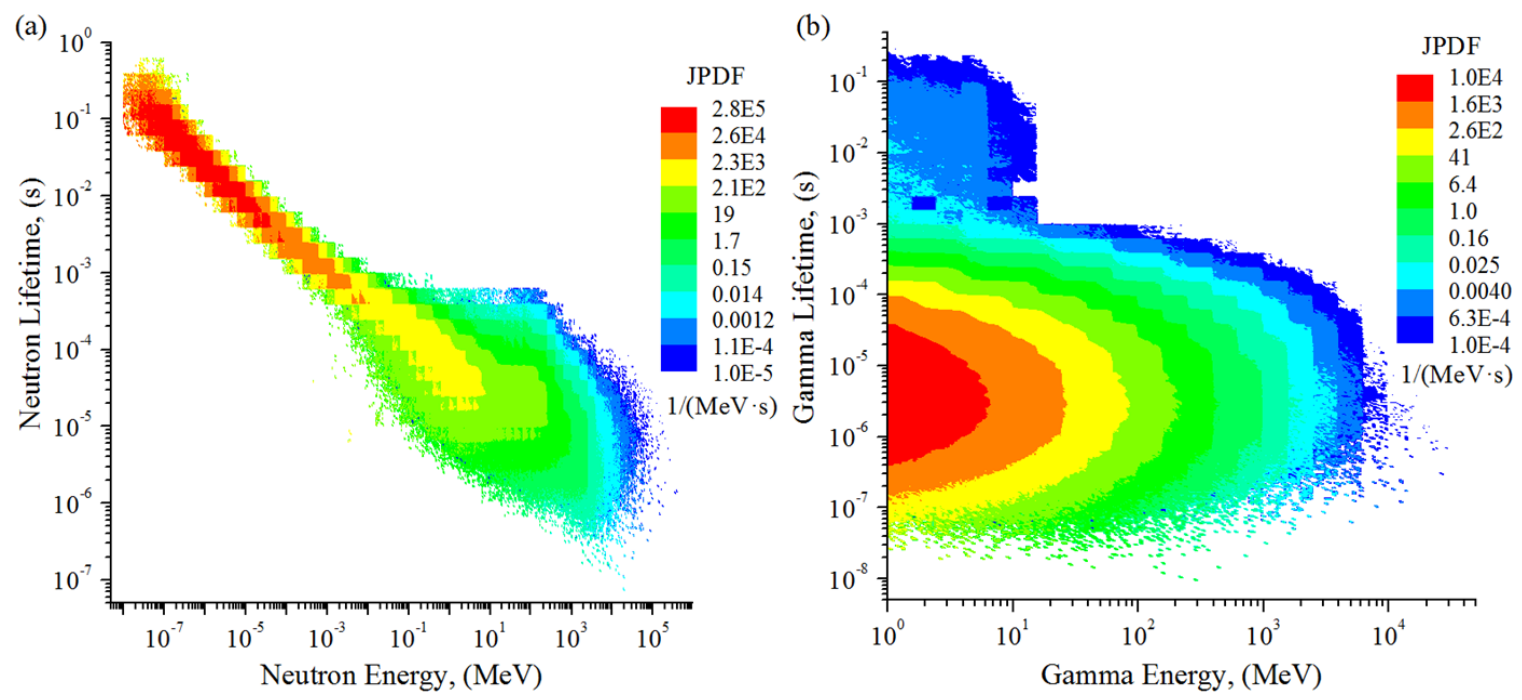

Fig. 7 
(a)

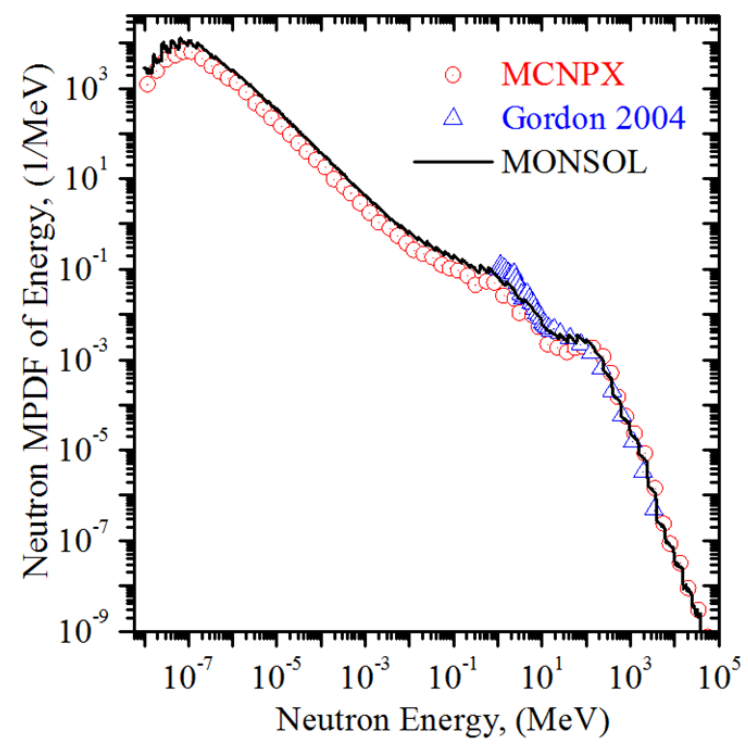

(b)

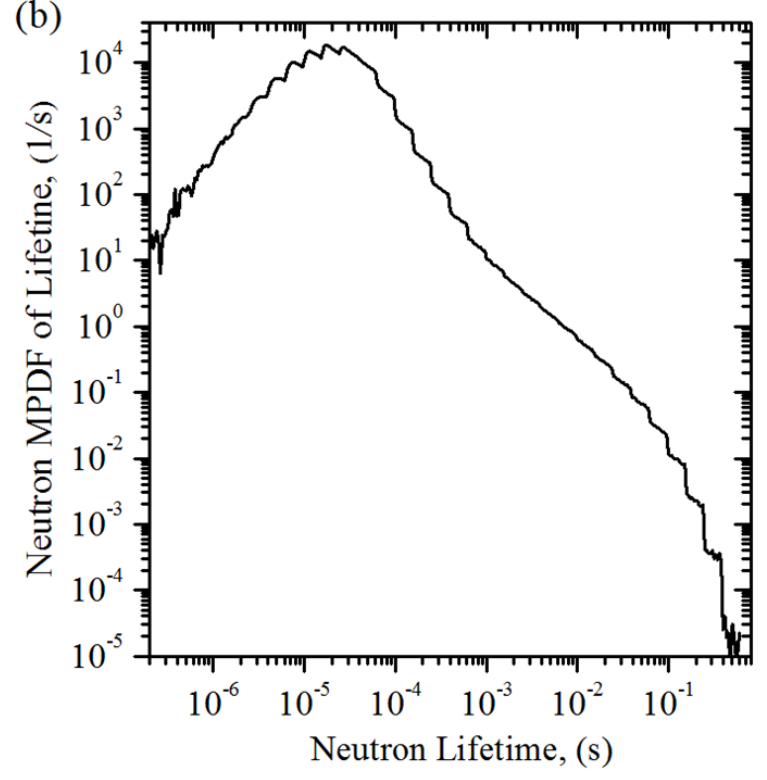

Fig. 8 

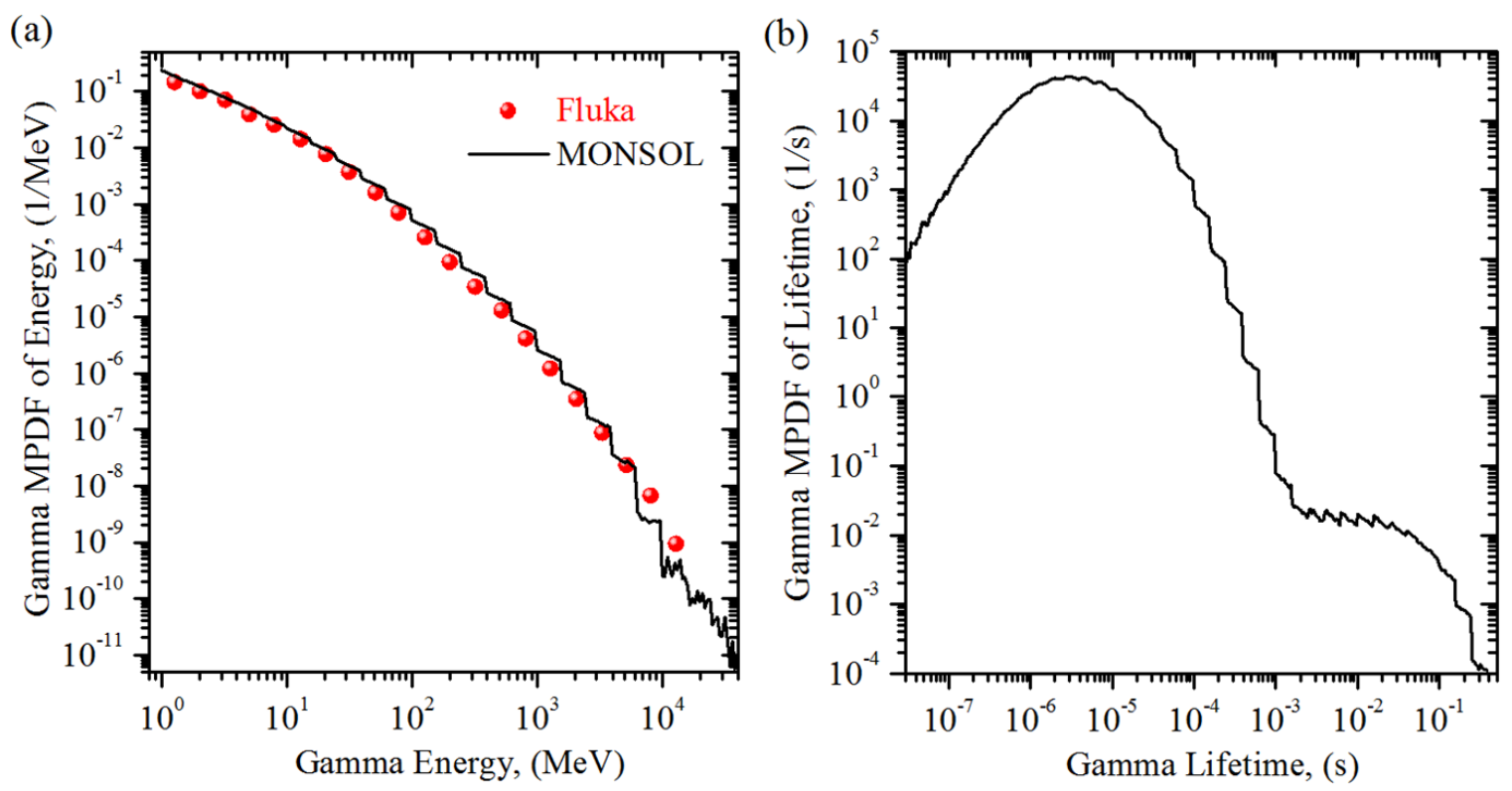

Fig. 9 


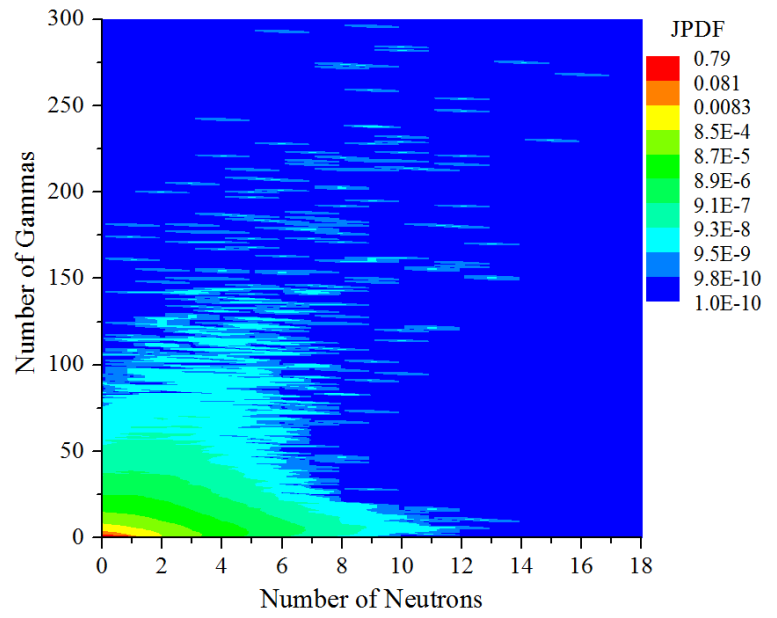

Fig. 10 

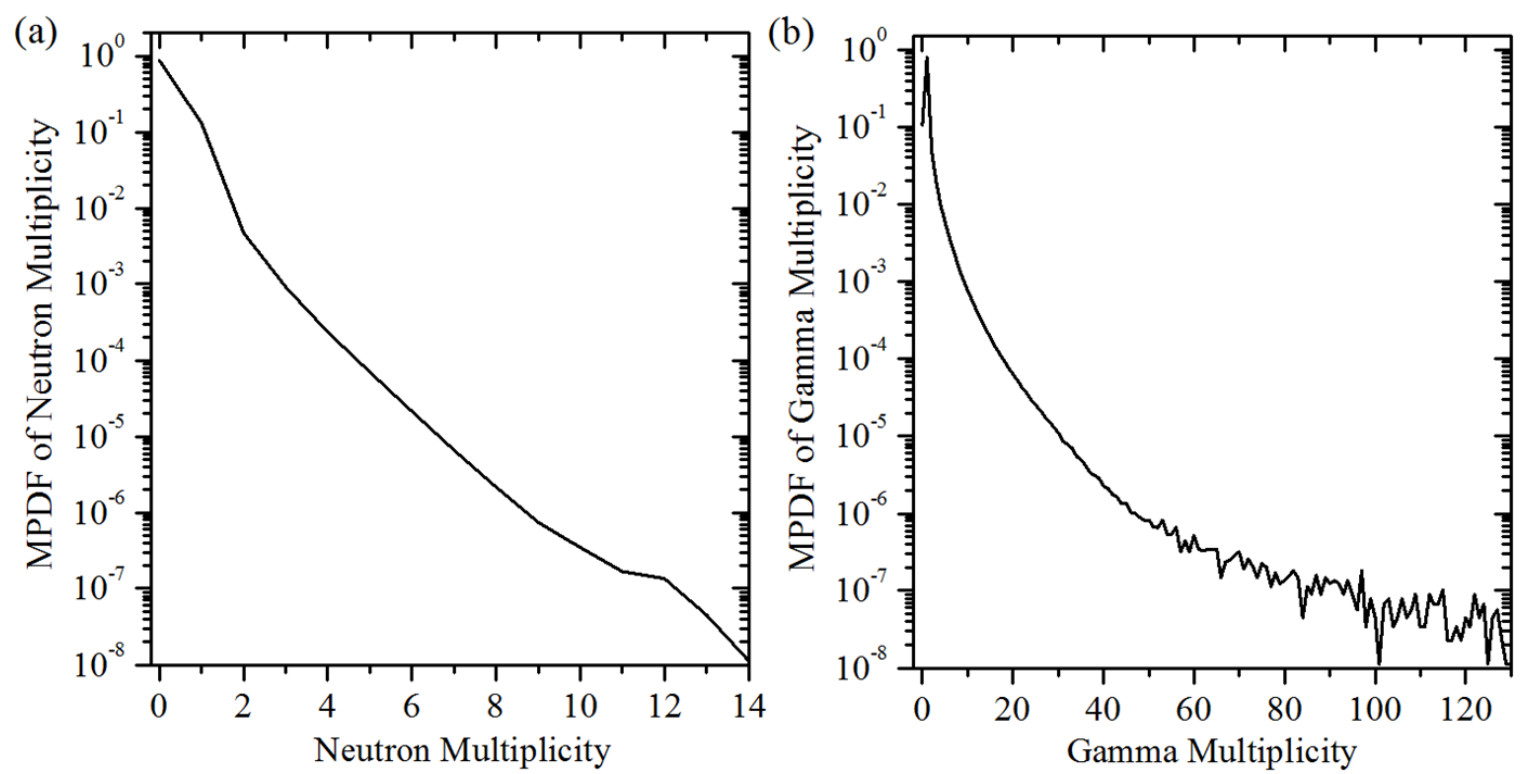

Fig. 11 


\begin{tabular}{lcclcc}
\hline & Energy (MeV) & Lifetime (ns) & Energy (MeV) & Lifetime (ms) \\
\hline SNM source & & \multicolumn{5}{c}{ non-SNM source } \\
Mean & $\sim 1.8$ & $\sim 2.9$ & Mean & $\sim 147$ & $\sim 0.58$ \\
Deviation & $\sim 1.4$ & $\sim 3.8$ & Deviation & $\sim 597$ & $\sim 6.1$ \\
& Energy-Lifetime & & Energy-Lifetime \\
\hline $\begin{array}{l}\text { Covariance } \\
\text { (MeV·ns) }\end{array}$ & $\sim-1.06$ & Covariance & $\sim-0.08$ \\
Correlation & & (MeV·s) & \\
\hline
\end{tabular}

Table 1 


\begin{tabular}{lcclcc}
\hline & Energy (MeV) & Lifetime (ns) & Energy (MeV) & Lifetime (ms) \\
\hline SNM source & \multicolumn{5}{c}{ non-SNM source } \\
Mean & $\sim 1.32$ & $\sim 2.77$ & Mean & $\sim 30$ & $\sim 0.1$ \\
Deviation & $\sim 0.92$ & $\sim 4.07$ & Deviation & $\sim 116$ & $\sim 3.1$ \\
& Energy-Lifetime & & Energy-Lifetime \\
\hline $\begin{array}{l}\text { Covariance } \\
\text { (MeV·ns) }\end{array}$ & & $\sim 0.068$ & Covariance & $\sim-0.002$ \\
Correlation & & (MeV·s) & $\sim-0.0055$ \\
\hline
\end{tabular}

Table 2 


\begin{tabular}{|c|c|c|c|c|c|}
\hline & Neutron & Gamma-rays & & Neutron & Gamma-rays \\
\hline SNM source & & & non-SNM source & & \\
\hline Mean & $\sim 2.6$ & $\sim 1.4$ & Mean & $\sim 0.14$ & $\sim 1.13$ \\
\hline Deviation & $\sim 1.6$ & $\sim 1.1$ & Deviation & $\sim 0.38$ & $\sim 1.23$ \\
\hline \multicolumn{4}{|c|}{ Neutron-Gamma-rays } & \multicolumn{2}{|c|}{ Neutron-Gamma-rays } \\
\hline Covariance & \multicolumn{2}{|c|}{$\sim 0.82$} & Covariance & \multicolumn{2}{|c|}{$\sim-0.021$} \\
\hline Correlation & \multicolumn{2}{|c|}{$\sim 0.48$} & Correlation & \multicolumn{2}{|c|}{$\sim-0.046$} \\
\hline
\end{tabular}

Table 3 


\section{References}

[1] J. Medalia, Detection of Nuclear Weapons and Materials: Science, Technologies, Observations, Prepared for Members and Committees of Congress June 4, 2010,

[2] R.C. Runkle, D.L. Chichester, S.J. Thompson, Rattling nucleons: New developments in active interrogation of special nuclear material, Nucl. Instrum. Methods Phys. Res., Sect. A, 663 (2012) 75-95.

[3] J. M. Verbeke, A. Dougan, L. F. Nakae, K. E. Sale, N.J. Snyderman, Neutron Correlations in Special Nuclear Materials, Experiments and Simulations, Proceedings of 48th INMM Annual Meeting, July 8, 2007 - July 12, 2007, Tucson, AZ, US,

[4] T. Gozani, Fission Signatures for Nuclear Material Detection, IEEE Trans. Nucl. Sci., 56 (2009) 736-741.

[5] N. Ensslin, W. C. Harker, M. S. Krick, D. G. Langner, M. M. Pickrell, J.E. Stewart, Application Guide to Neutron Multiplicity Counting, LA-13422-M Manual UC-700, 1998,

[6] S. Fetter, V. A. Frolov, M. Miller, R. Mozley, O. F. Prilutsky, S. N. Rodionov, R.Z. Sagdeev, Detecting Nuclear Warheads, Science \& Global Security, Vol. 1, No. 3-4, 1990, pp. 225-253,

[7] J. M. Verbeke, C. Hagmann, D. Wright, Simulation of Neutron and Gamma Ray Emission from Fission, UCRL-AR-228518, Lawrence Livermore National Laboratory (2009),

[8] M. Owen, G. Weston, J. O'Malley, AWE development of active interrogation techniques for the detection of SNM, Proc Spie, 7304 (2009). 
[9] G.W. Phillips, D.J. Nagel, T. Coffey, A Primer on the Detection of Nuclear and Radiological Weapons, Washington, DC: Center for Technology and National Security Policy, May 2005,

[10] E. Aguayo, R. T. Kouzes, A. S. Ankney, J. L. Orrell, T. J. Berguson, M.D. Troy, Cosmic Ray Interactions in Shielding Materials, Pacific Northwest National Laboratory Report No. PNNL-20693, 2011,

[11] P.K.F. Grieder, Cosmic rays at Earth: researcher's reference manual and data book, 1st ed., Elsevier Science Ltd., Amsterdam; New York, 2001.

[12] R.T. Kouzes, J.H. Ely, A. Seifert, E.R. Siciliano, D.R. Weller, L.K. Windsor, M.L. Woodring, J. Borgardt, E. Buckley, E. Flumerfelt, A. Oliveri, M. Salvitti, Cosmic-ray-induced ship-effect neutron measurements and implications for cargo scanning at borders, Nucl. Instrum. Methods Phys. Res., Sect. A, 587 (2008) 89-100.

[13] T. Gozani, D. Strellis, Advances in neutron based bulk explosive detection, Nucl Instrum Meth B, 261 (2007) 311-315.

[14] A. Enqvist, S.A. Pozzi, I. Pazsit, The detection statistics of neutrons and photons emitted from a fissile sample, Nucl. Instrum. Methods Phys. Res., Sect. A, 607 (2009) 451-457.

[15] G. Miloshevsky, A. Hassanein, Time correlation of cosmic-ray-induced neutrons and gamma rays at sea level, Nucl Instrum Meth A, 737 (2014) 33-41.

[16] G. Miloshevsky, A. Hassanein, Energy-angle correlation of neutrons and gamma rays emitted from an HEU source, Nucl Instrum Meth A, 749 (2014) 47-56. 
[17] A. J. Koning, S. Hilaire, M.C. Duijvestijn, TALYS-1.0, Proceedings of the International Conference on Nuclear Data for Science and Technology - ND2007, April 22-27, 2007, Nice, France, eds. O. Bersillon, F. Gunsing, E. Bauge, R. Jacqmin and S. Leray, EDP Sciences, 2008, p. 211-214.,

[18] http://www.talys.eu/documentation/.

[19] C. Hagmann, D. Lange, D. Wright, Cosmic-ray shower generator (CRY) for Monte Carlo transport codes, Nuclear Science Symposium Conference Record, 2007. , Oct. 26 2007-Nov. 3 2007, 2007

[20] K. Ide, M.F. Becchetti, M. Flaska, A. Poitrasson-Riviere, M.C. Hamel, J.K. Polack, C.C. Lawrence, S.D. Clarke, S.A. Pozzi, Analysis of a measured neutron background below $6 \mathrm{MeV}$ for fast-neutron imaging systems, Nucl. Instrum. Methods Phys. Res., Sect. A, 694 (2012) 24-31.

[21] S.A. Pozzi, E. Padovani, M. Marseguerra, MCNP-PoliMi: a Monte-Carlo code for correlation measurements, Nucl. Instrum. Methods Phys. Res., Sect. A, 513 (2003) 550-558.

[22] M.S. Gordon, R. Goldhagen, K.P. Rodbell, T.H. Zabel, H.H.K. Tang, J.M. Clem, P. Bailey, Measurement of the flux and energy spectrum of cosmic-ray induced neutrons on the ground, IEEE Trans. Nucl. Sci., 51 (2004) 3427-3434. 